\title{
Progress and Challenges in the Theory of Nuclei ${ }^{\star}$
}

\author{
D.J. Dean \\ Physics Division, Oak Ridge National Laboratory, P.O. Box 2008, Oak Ridge, TN 37831-6373 USA
}

\begin{abstract}
Nuclear theory today aims for a comprehensive theoretical framework that can describe all nuclei. I discuss recent progress in this pursuit and the associated challenges as we move forward.

Key words: nuclear theory, many-body theory

PACS: 24.10.Cn
\end{abstract}

\section{The journey toward a comprehensive theory of nuclei}

Nuclei comprise $99.9 \%$ of all baryonic matter in the Universe and are the fuel that burns in stars 11. The rather complex nature of the nuclear forces among protons and neutrons generates a broad range and diversity in the nuclear phenomena that we observe. Experiments indicate that developing a comprehensive description of all nuclei and their reactions requires theoretical and experimental investigations of rare isotopes with unusual neutron-to-proton ratios that are very different from their stable counterparts. These rare nuclei are difficult to produce and study experimentally since they can have extremely short lifetimes. The goal of a comprehensive description and reliable modeling of nuclei represents one of the great intellectual opportunities for nuclear physics in the coming years.

Key scientific themes being addressed by the physics of nuclei and nuclear astrophysics communities are captured by five overarching questions:

- What is the nature of the nuclear force that binds protons and neutrons into stable nuclei and rare isotopes?

- What is the origin of simple patterns in complex nuclei?

- What is the nature of neutron stars and dense nuclear matter?

ऋ Oak Ridge National Laboratory is managed by UT-Battelle for the U.S. Department of Energy under Contract No. DE-AC05-00OR22725. 
- What is the origin of the elements in the cosmos?

- What are the nuclear reactions that drive stars and stellar explosions?

These questions align well with the drivers of rare isotope science as recently summarized in a report of the U. S. National Academies of Science Rare Isotope Scientific Assessment Committee (RISAC) 22. One primary aspect of the first and second question concerns testing the predictive power of models by extending experiments to new regions of mass and proton-to-neutron ratio and identifying new phenomena that will challenge existing many-body theory.

One of those challenges concerns the concept of nuclear shell structure and magic numbers. Understanding the origin of the classic, stable, nuclear magic numbers earned Goeppert-Mayer and Jensen the 1963 Nobel Prize. At the time, there were no data on very neutron-rich nuclei; however, theoretical predictions and experimental discoveries in the last decade indicate that nuclear shell structure is a rather fluid concept. For example, experimental data indicate that the magic numbers at $\mathrm{N}=20$ and 28 fade away with neutron number, and the new magic numbers at $\mathrm{N}=14,16$, and 32 seem to appear (see, for example Ref. [3]). Nuclei far from stability also exhibit unusual properties as compared to their stable cousins. For example, the radial extension of the two-neutron halo in ${ }^{11} \mathrm{Li}$ (where ${ }^{9} \mathrm{Li}$ acts as a core) is the same as that of ${ }^{208} \mathrm{~Pb}$.

We can conclude from just these two examples that nuclear structure is changing in the exotic environment. Since nuclei are self-bound, they generate their own mean field which determines how shell orbitals are filled. The experimental evidence indicates the way orbits are being filled is changing in neutron-rich nuclei. Furthermore, manybody correlations such as pairing superfluidity become crucial when the binding energy becomes small. These correlations also affect the open-shell character of nuclei, since the continuum of scattering states lies very close to the bound state.

One of the interesting features of nuclear physics today is the overlaps and synergy between the physics of nuclei and nuclear astrophysics. The changes in nuclear structure occurring away from the valley of beta-stability do affect nucleosynthesis pathways. We have initial evidence of this on the measurements of the lifetime of ${ }^{78} \mathrm{Ni}$ [4] and its impact on r-process nucleosynthesis. Other examples involve the impact of electron capture on the core collapse mechanism of type-II supernovae [5] and the challenge of obtaining a nuclear equation of state that can quantitatively be used in predictions of neutron star properties 6 .

A robust theoretical capability is required if we want to understand stable and exotic nuclei that are the core of matter and the fuel of stars. I will devote the remainder of this Proceedings to a discussion of some of the issues that remain in building this capability.

\section{Progress and challenges in the theory of nuclei}

The nuclear many-body problem spans nuclei from $A=2$ (the deuteron) to the superheavy region, and different theoretical techniques are pursued in different regions of the chart of nuclei. This is depicted in Fig. 1 where the current reach of various theoretical methods is interposed in the chart of nuclei. The challenges for theory can be viewed broadly as: determining the nuclear interaction, calculating ab initio nuclei, including the continuum, and finding an appropriate energy density functional for a nuclear Density Functional Theory approach. I will briefly discuss these areas in the following subsections, 


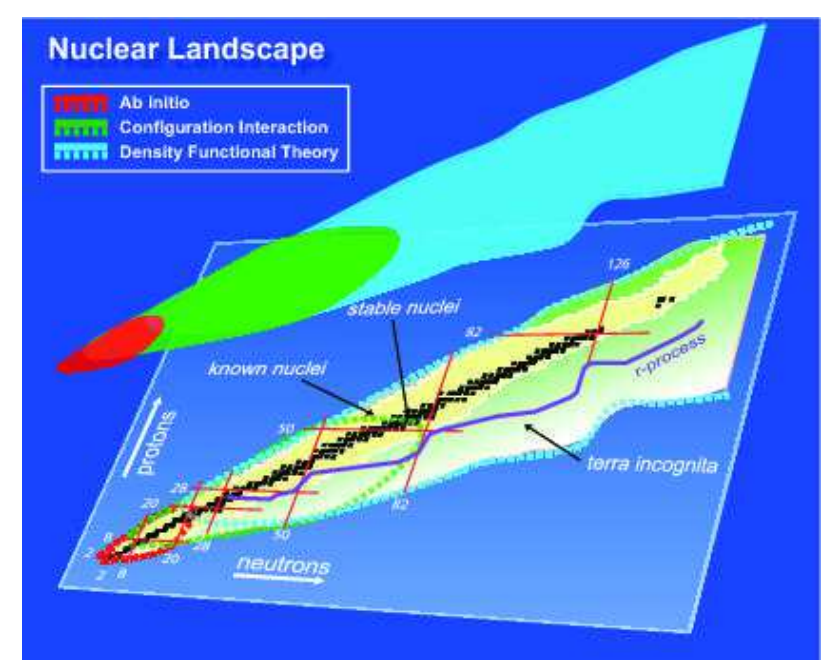

Fig. 1. (Color online) The theoretical methods and computational techniques used to solve the nuclear many-body problem. The red vertical and horizontal lines show the magic numbers, reflecting regions where nuclei are expected to be more tightly bound and have longer half-lives. The anticipated path of the astrophysical r-process responsible for nucleosynthesis of heavy elements is also shown (purple line). The thick dotted lines indicate domains of major theoretical approaches to the nuclear many-body problem. For the lightest nuclei, ab initio calculations (Green's function Monte Carlo, no-core shell model, coupled cluster method), based on the bare nucleon-nucleon interaction, are possible (red). Medium-mass nuclei can be treated by configuration interaction techniques [interacting shell model (green)]. For heavy nuclei, the density functional theory based on self-consistent/mean-field theory (blue) is the tool of choice. By investigating the intersections between these theoretical strategies, one aims to develop a unified description of the nucleus.

drawing from some of the work in which I have been involved.

\subsection{Determining nuclear interactions}

The forces among nucleons determine how nuclei behave. These forces arise from QCD. While there is some effort to extract directly from lattice calculations 7 the nuclear scattering, there have also been important developments in the application of a chiral Effective Field Theory (EFT) to the nuclear problem [89. An EFT was developed for nuclei from a chiral Lagrangian that maintains all the symmetries of QCD and treats as fundamental particles the pions and nucleons. A power-counting scheme was developed so that the expansion could be carried out in a systematic way using as a small parameter of expansion the momentum transfer in nucleon-nucleon scattering divided by the QCD energy scale $(1 \mathrm{GeV})$. This expansion has been carried out to fourth-order (known as $\mathrm{N}^{3} \mathrm{LO}$ ) [10. The resulting nucleon-nucleon potential fits all scattering phase shifts up to the cut-off used in the EFT.

An interesting feature of the EFT is that three-nucleon interactions arise naturally at the third order $\left(\mathrm{N}^{2} \mathrm{LO}\right)$. Chiral three-body forces have been known for a long time, but their systematic computation was not known until recently. Nuclear spectroscopy using three-body interactions from the EFT expansion improve when compared to calculations with two-body forces alone [11. This is also to be expected since it has long been known 
that two-body forces alone do not reproduce the correct binding in ${ }^{4} \mathrm{He}$.

Thus, our starting point is a Hamiltonian of the form

$$
H=t-t_{c m}+V_{2}+V_{3},
$$

where $t$ is the kinetic energy operator and $t_{c m}$ is the center-of-mass operator (so that the kinetic energy maintains translational invariance) and the $V_{2}$ and $V_{3}$ represent the two-body and three-body nuclear potentials. The three-body interaction can actually be written in normal-ordered form as

$$
V_{3}=\frac{1}{6} \sum_{i j k}\langle i j k \| i j k\rangle+\frac{1}{2} \sum_{i j p q}\langle i j p \| i j q\rangle\left\{\hat{a}_{p}^{\dagger} \hat{a}_{q}\right\}+\frac{1}{4} \sum_{i p q r s}\langle i p q \| i r s\rangle\left\{\hat{a}_{p}^{\dagger} \hat{a}_{q}^{\dagger} \hat{a}_{s} \hat{a}_{r}\right\}+\hat{h}_{3},
$$

where $\langle\alpha \beta \gamma \| \delta \epsilon \eta\rangle$ represents a matrix element of the three-body interaction, $i, j, k$ denote the hole states, $a, b, c$ denote the particle states, $p, q, r$ denote all states, and $\hat{h}_{3}$ denotes the residual three-body Hamiltonian

$$
\hat{h}_{3} \equiv \frac{1}{36} \sum_{p q r s t u}\langle p q r \| s t u\rangle\left\{\hat{a}_{p}^{\dagger} \hat{a}_{q}^{\dagger} \hat{a}_{r}^{\dagger} \hat{a}_{u} \hat{a}_{t} \hat{a}_{s}\right\} .
$$

This notation will be useful in what follows.

$V_{2}$ is often modeled with a very repulsive short distance interaction. In the case of Av18 [12, the central repulsion at $r=0$ is approximately $3 \mathrm{GeV}$; in $\mathrm{N}^{3} \mathrm{LO}$, it is 500 $\mathrm{MeV}$ [10. In order to use these interactions efficiently in a framework where one expands the many-body wave function in a basis, it is desirable to renormalize these interactions. This can be done in several ways, including the $G$-matrix approach [13] and similarity transform methods 14 .

More recently, work in the direction of renormalization group (RG) [15]16 techniques has been used to evolve the nucleon-nucleon interactions from high momentum to low momentum while preserving the nucleon-nucleon phase shifts up a given momentum. These interactions can then be projected onto the appropriate basis states for computation. These approaches offer promise with a price: the resulting two-body interactions do not recover the binding energy that would be obtained from a pure two-body calculation, and one must indeed include a three-body force in the calculations. Furthermore, the RG interactions tend toward overbinding both in nuclei $[17$ and in nuclear matter $[18$. The cure to this problem is the introduction of three-body forces which naturally occur in the RG approaches. Another possibility is to adjust the three-body force that comes from EFT to obtain the experimental binding of ${ }^{4} \mathrm{He}[19]$.

\subsection{Calculating ab initio Nuclei}

For light nuclei with mass numbers $A \leq 12$, both Green's function Monte Carlo methods [20] and no-core shell-model calculations [21/11] using a basis-state expansion provide almost converged benchmarks for selected two- and three-body Hamiltonians. The agreement with experimental data for many light nuclei is quite reasonable in these calculations. GFMC techniques do not need to utilize renormalized interactions, but they can only use local potentials, and the main group involved uses one two-body potential $($ Av18, [12]) and an appropriate three-body potential fitted to experimental data in light 
nuclei 22. The NCSM approach can use any potential, but in most cases uses renormalized ones in order to obtain converging calculations. Both methods exhibit exponential scaling with the number of particles (to reach the same accuracy as in ${ }^{4} \mathrm{He}$ with the same methods). Recently, we have seen a movement of these methods into the realm of light nuclear reactions. For example, the GFMC approach was used to describe $n+\alpha$ scattering [23] while the NCSM was used to calculate the ${ }^{7} \mathrm{Be}(p, \gamma)^{8} \mathrm{~B}$ S-factor [24]. Recent progress has also been made in ab initio inelastic four-body scattering problems [25].

Coupled-cluster theory [26/27/28/29] represents another approach to ab initio calculations of nuclei. Along with collaborators, including computational chemists, we have developed the theory and methods $30|31| 32|33| 17$ to the point where we can calculate nuclear properties in medium-mass nuclei. Nuclear coupled-cluster theory exhibits polynomial scaling with the size of the basis state and number of particles, and is sizeextensive. This very important property means that no unlinked diagrams enter into the theory.

Coupled-cluster theory starts with the simple assertion that the correlated groundstate wave function can be described by applying an exponentiated correlation operator to an uncorrelated Slater determinant that naively describes the nucleus:

$$
|\Psi\rangle=\exp (T)|\Phi\rangle \text {. }
$$

The correlation operator $T$ induces various $n \mathrm{p}-n \mathrm{~h}$ (particle-hole) correlations (up to the number of nucleons, $A$, in the nucleus):

$$
T=T_{1}+T_{2}+\cdots T_{A} .
$$

The energy of the ground state is given by

$$
E=\langle\Phi|\exp (-T) H \exp (T)| \Phi\rangle=\langle\Phi|\bar{H}| \Phi\rangle=\left\langle\Phi\left|(H \exp (T))_{c}\right| \Phi\right\rangle,
$$

where the subscript $c$ means that only connected diagrams enter into the expressions. The $T_{n}$ operators take the form (as is the case here for the two-body excitation operator)

$$
T_{2}=\sum_{a b, i j} t_{i j}^{a b} a_{a}^{\dagger} a_{b}^{\dagger} a_{j} a_{i}
$$

where $t_{i j}^{a b}$ are the $2 p-2 h$ correlation amplitudes.

One must compute the correlation amplitudes through equations that left-project $\bar{H}$ onto the space of excited Slater determinants. For example, if the theory only contains $T_{1}$ and $T_{2}$ operators, then the equations to solve for the coefficients $t_{i}^{a}$ and $t_{i j}^{a b}$ are

$$
\begin{aligned}
& 0=\left\langle\Phi_{i}^{a}|\bar{H}| \Phi\right\rangle \\
& 0=\left\langle\Phi_{i j}^{a b}|\bar{H}| \Phi\right\rangle,
\end{aligned}
$$

and so on.

Eqn. (6) is exact; however, the power of the coupled-cluster theory is its highly accurate computation of the energy even when one limits to lower order the number of $T_{n}$ operators in the theory. For example, at the $T_{1}$ and $T_{2}$ levels (coupled clusters in singles and doubles, or CCSD), approximately $90 \%$ of the correlation is obtained, while with approximate triples corrections (denoted $\operatorname{CCSD}(\mathrm{T})$ ), nearly all of the correlation energy is obtained 
Table 1

\begin{tabular}{l|r|r|r}
\hline & \multicolumn{1}{|c|}{${ }^{4} \mathrm{He}$} & \multicolumn{1}{c|}{${ }^{16} \mathrm{O}$} & ${ }^{40} \mathrm{Ca}$ \\
\hline \hline$E_{0}$ & -11.8 & -60.2 & -347.5 \\
$\Delta E_{\mathrm{CCSD}}$ & -17.1 & -82.6 & -143.7 \\
$\Delta E_{\mathrm{CCSD}(\mathrm{T})}$ & -0.3 & -5.4 & -11.7 \\
\hline$E_{\mathrm{CCSD}(\mathrm{T})}$ & -29.2 & -148.2 & -502.9 \\
\hline \hline exact (FY) & $-29.19(5)$ & & \\
\hline
\end{tabular}

Reference vacuum energies, $E_{0}, \mathrm{CCSD}$ and $\operatorname{CCSD}(\mathrm{T})$ correlation energies, $\Delta E_{\mathrm{CCSD}}$ and $\Delta E_{\mathrm{CCSD}(\mathrm{T})}$, and binding energies $E_{\mathrm{CCSD}(\mathrm{T})}$ for ${ }^{4} \mathrm{He},{ }^{16} \mathrm{O}$ and ${ }^{40} \mathrm{Ca}$. The vacuum energies, $E_{0}$, are for $\hbar \omega=14 \mathrm{MeV}$ in the case of ${ }^{4} \mathrm{He}$ and $\hbar \omega=22 \mathrm{MeV}$ for ${ }^{16} \mathrm{O}$ and ${ }^{40} \mathrm{Ca}$. The CCSD and CCSD(T) energies are the extrapolated infinite model space results. The exact Faddeev-Yakubovsky result is from Ref. [19]

3117. This contrasts sharply to truncated shell-model calculations where the truncation introduces unlinked diagrams causing growing errors with particle number 34.

A certain amount of benchmarking of methods is necessary in order to understand their regions of validity. For coupled-cluster theory, this has recently been performed in the triton and ${ }^{4} \mathrm{He}$. We also performed extremely large basis-set calculations to which other methods can compare when they reach the technical capability to do so [17. We summarize our coupled-cluster results for the binding energies of ${ }^{4} \mathrm{He},{ }^{16} \mathrm{O}$, and ${ }^{40} \mathrm{Ca}$ in Table 1, which gives the extrapolated correlation energies $\Delta E_{\mathrm{CCSD}}$ and $\Delta E_{\mathrm{CCSD}(\mathrm{T})}$, relative to the uncorrelated energy $E_{0}=\langle\Phi|H| \Phi\rangle$. We find that for ${ }^{4} \mathrm{He},{ }^{16} \mathrm{O}$, and ${ }^{40} \mathrm{Ca}$, the triples corrections are a factor of $\approx 0.015,0.066$, and 0.081 smaller than the CCSD correlation energies. From this, we estimate the missing correlation energy from quadruples, pentuplets, and so on to be of the order of $1 \mathrm{MeV}$ for ${ }^{40} \mathrm{Ca}$. We note that ${ }^{16} \mathrm{O}$ is overbound by about $20 \mathrm{MeV}$, and ${ }^{40} \mathrm{Ca}$ by about $150 \mathrm{MeV}$ when compared to the experimental binding energies. This is not surprising and points to the importance of $3 \mathrm{NF}$ for nuclear structure calculations and to its apparently large role in the RG prescriptions.

The three-body force is a key component of modern nuclear Hamiltonians. Other methods have been adapted to include three-body interactions already several years ago. We recently developed coupled-cluster theory for three-body forces 33 . This represents a major improvement to the scientific reach of modern coupled-cluster calculations for nuclei. An exponential extrapolation of the (approximate) $\operatorname{CCSD}(\mathrm{T})$ minima to an infinite model space is shown in Fig. 2 and yields $E_{\infty}=-28.24 \mathrm{MeV}$. This is in excellent agreement with the exact Faddeev-Yakubovsky result $E=-28.20(5) \mathrm{MeV}$. In our largest model space at the minimum $\hbar \omega=17 \mathrm{MeV}$, the ground-state expectation values for the center-of-mass Hamiltonian is $\left\langle H_{\mathrm{cm}}\right\rangle \approx 20 \mathrm{keV}$ while the expectation value for the angular momentum is zero for a closed-shell nucleus by construction.

It is interesting to analyze the different contributions $\Delta E$ to the binding energy $E$. The individual contributions are given in Fig. 2 for a model space of $N=4$ oscillator shells and $\hbar \omega=20 \mathrm{MeV}$. The main contribution stems from the low-momentum NN interaction. The contributions from 3NFs account only for about $10 \%$ of the total binding energy. This is consistent with the chiral EFT power-counting estimate $\left\langle V_{3 \mathrm{~N}}\right\rangle \sim$ $\left(Q / \Lambda_{\chi}\right)^{3}\left\langle V_{\text {lowk } k}\right\rangle \approx 0.1\left\langle V_{\text {low } k}\right\rangle[19$. The second-, third-, and fourth-largest contribution are due to the first, second, and third term on the right-hand side of Eq. (2). These are the density-dependent zero-, one-, and two-body terms, which resulted from the normal ordering of the three-body Hamiltonian in coupled-cluster theory. The contributions from 

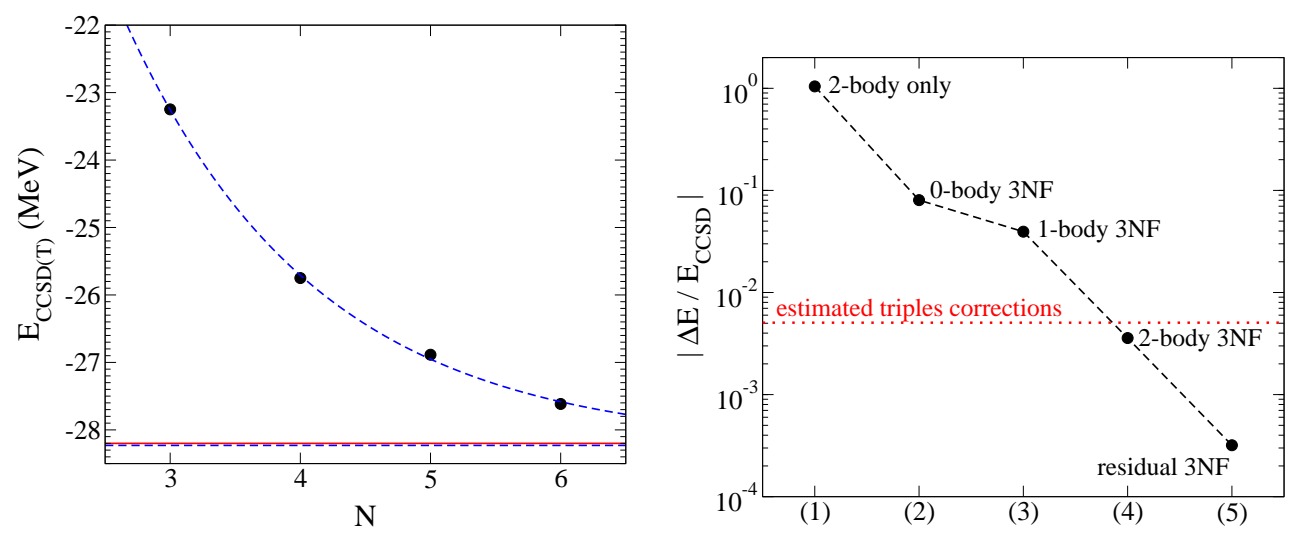

Fig. 2. Left: (Color online) Data points: $\operatorname{CCSD}(\mathrm{T})$ results (taken at the $\hbar \omega$ minima) for the binding energy of ${ }^{4} \mathrm{He}$ with $3 \mathrm{NFs}$ as a function of the number of oscillator shells. Dashed lines: Exponential fit to the data and asymptote of the fit. Full line: Exact result. Right: Relative contributions $|\Delta E / E|$ to the binding energy of ${ }^{4} \mathrm{He}$ at the CCSD level. The different points denote the contributions from (1) low-momentum NN interactions, (2) the vacuum expectation value of the $3 \mathrm{NF}$, (3) the normal-ordered one-body Hamiltonian due to the $3 \mathrm{NF}$, (4) the normal-ordered two-body Hamiltonian due to the $3 \mathrm{NF}$, and (5) the residual 3NFs. The dotted line estimates the corrections due to omitted three-particle-three-hole clusters.

the residual three-body Hamiltonian, Eq. (3), are very small and are represented by the last point in the right panel of Fig. 2. The residual 3NF contributes to the energy directly and indirectly through a modification of the $T_{1}$ and $T_{2}$ cluster amplitudes. Apparently, both contributions are very small. This is a promising result, and time will tell whether it holds for heavier nuclei.

\subsection{Presence of the continuum}

Exotic phenomena emerge in weakly bound and resonant many-body quantum systems. These phenomena include ground states that are embedded in the continuum, melting and reorganizing of shell structures, extreme matter clusters and halo densities. A theoretical description of weakly bound and unbound quantum many-body systems represents a challenging undertaking. The weakly bound character of these systems means that they should be treated as open quantum systems where coupling with the scattering continuum can take place. Furthermore, theoretical treatments that consider continuum basis states can describe resonant widths (lifetimes).

Recent work with Gamow states employed in Hamiltonian diagonalization methods 35136 37. have shown that these basis states correctly depict properties associated with open quantum systems. This Berggren basis is composed of bound, resonant, and (continuum) scattering single-particle states 38 . This basis significantly improves and facilitates the description of loosely bound systems and is essential in the description of unbound systems. In addition, several groups have worked on alternative methods, such as the continuum shell model [39,40] and the recently developed shell model embedded in the continuum 41.

We recently developed a complex version of the coupled-cluster method that can utilize the Gamow-basis to calculate widths of states in the He isotopic chain in an ab initio 


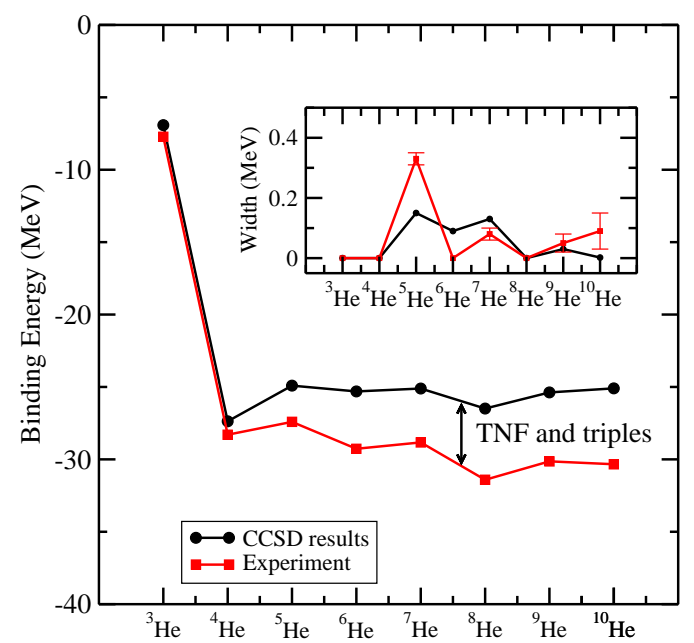

Fig. 3. (Color online) CCSD calculation of the ${ }^{3-10} \mathrm{He}$ ground states with the low-momentum $\mathrm{N}^{3} \mathrm{LO}$ nucleon-nucleon interaction for increasing number of partial waves. The energies $E$ are given in $\mathrm{MeV}$ for both real and imaginary parts. Experimental data are from Ref. 42 . Our calculated width of ${ }^{10} \mathrm{He}$ is $\approx$ $0.002 \mathrm{MeV}$.

framework 32. We utilized a $\mathrm{V}_{\text {low } k}$ interaction from $\mathrm{N}^{3} \mathrm{LO}$ at a cut-off of $1.9 \mathrm{fm}^{-1}$ in a Berggren basis to perform the calculations. These novel calculations, the results of which are shown in Fig. 3, while by no means perfect, utilizing only a two-body interaction, do indicate the power of moving beyond the shell model.

\subsection{Density functional theory for nuclei}

Nuclear Density Functional Theory (nDFT) is being used and improved to describe heavier nuclei. The basic idea is to expand the energy density functional to second order (gradient terms) in the density and to calculate with this functional all nuclear properties. One must determine the coupling constants that characterize the functional from experimental data. Odd- $A$ and odd-odd nuclei will be key to any new parameterizations as they allow one to interrogate time-odd terms in the energy density functionals.

The nuclear DFT efforts have been quite successful in describing a wide variety of nuclear data with very good precision across the nuclear chart. The various parameterizations usually work quite well in regions where nuclear masses and other properties are experimentally determined, but extrapolations into very neutron-rich nuclei have been problematic. The next-generation experimental facilities should enable theorists to obtain a functional parameterization that will describe bulk properties of all nuclei. Various nuclear data along long isotopic and isotonic chains are needed to constrain the isovector part of the energy functional. More specifically, one needs (difference of) masses and measures of collectivity and of the shell evolution in unknown regions, where predictions of currently used functionals disagree. Initial attempts are represented in Fig. 4 where the two-neutron separation energies were calculated from an even-even mass table for the Sly4 Skyrme interaction with pairing included and partial number projection 43 . Other groups are also investigating nuclear mass systematics in large mass-table calculations 


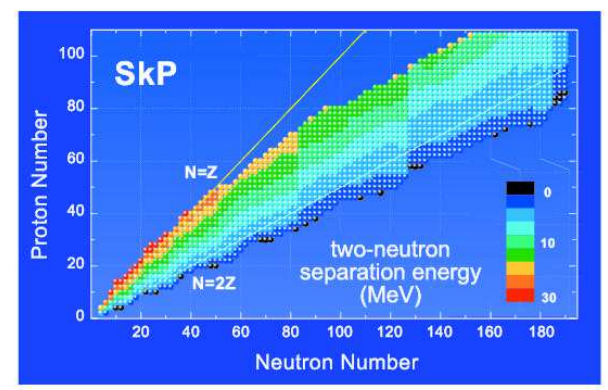

Fig. 4. (Color online) An example of a large-scale systematic density functional theory (DFT) calculation for complex nuclei: results of the deformed DFT calculations of two-neutron separation energies for 1553 particle-bound even-even nuclei with $Z \leq 108$ and $N \leq 188$ (from M. Stoitsov).

and have recently extended these calculations to include a study of fission barriers [44]. Other data that could be included in determining the coupling constants of the energy density functional, such as large deformations (at low and high angular momentum) and multipole strength distributions in neutron-rich nuclei, will also be extremely valuable. The quest for a universal energy density function represents one of the key challenges of a U.S. Department of Energy SciDAC project.

\section{The next 10 years}

I believe that the next 10 years hold great promise for the physics of nuclei and for moving toward the ultimate solution of the nuclear problem: to obtain a comprehensive understanding of all nuclei and their interactions. On the experimental side, new facilities at RIKEN, GSI, and GANIL, and the future FRIB in the U.S. will make the discoveries that will challenge our theories and our understanding of many-body phenomena.

From a theoretical point of view, I believe the key to reaching the goal is the pursuit of many-body theory and its connections to nuclear DFT. We will see in the next few years the development of nuclear theory such that robust and predictive ab initio calculations can be accomplished in medium-mass nuclei. The effort to establish an improved density functional should allow for breakthrough science in heavy nuclei and in nuclei along the various nucleosynthesis paths. The strides being made to connect ab initio calculations in light- to medium-mass systems to nuclear DFT will enable us to finally rest the energy density functional on a firmer footing. The links from QCD to the underlying interaction will allow us to close the loop and reliably compute nuclei from the ground up.

All of the efforts I described in the preceding section - GFMC, NCSM, coupled-cluster efforts, and the quest to find the universal nuclear energy density functional - require petascale computational capability. We have witnessed in the last five years tremendous growth in computing capability and many in our community continue to embrace these marvelous tools. The ingenuity of researchers to utilize the largest of these machines undoubtedly will propel the field forward in our quest to understand nuclei. 


\section{References}

[1] Nuclear Physics: The core of matter, the fuel of stars, National Academy Press, Washington, DC (1999)

[2] Scientific Opportunities with a Rare-Isotope Facility in the United States, National Academies Press, Washington, DC (2007)

[3] J. Fridmann et al., Nature 435, 992 (2005)

[4] P.T. Hosmer et al., Phys. Rev. Lett. 94, 112501 (2005)

[5] K. Langanke, G. Martinez-Pinedo, J.M. Sampaio, D.J. Dean, W.R. Hix, O.E. Messer, A. Mezzacappa, M. Liebendörfer, H.-Th. Janka, and M. Rampp, Phys. Rev. Lett. 90, 241102 (2003)

[6] P. Gögelein and H. Müther, Phys. Rev. C 76, 024312 (2007)

[7] N. Ishii, S. Aoki, T. Hatsuda, Phys. Rev. Lett. (in press, 2007), and arXiv:nucl-th/0611096

[8] S. Weinberg, Phys. Lett. B 251, 288 (1990)

[9] C. Ordónez, L. Ray, and U. van Kolck, Phys. Rev. Lett. 72, 1982 (1994)

[10] D.R. Entem and R. Machleidt, Phys. Rev. C 68, 041001(R) (2003)

[11] P. Navratil, V.G. Gueorguiev, J.P. Vary, W.E. Ormand, and A. Nogga, Phys. Rev. Lett. 99, 042501 (2007)

[12] R.B. Wiringa, V.G.J. Stoks, and R. Schiavilla, Phys. Rev. C 51, 38 (1995)

[13] M. Hjorth-Jensen, T.T.S. Kuo, and E. Osnes, Phys. Repts. 261, 125 (1995)

[14] S.Y. Lee and K. Suzuki, Phys. Lett. B 91, 79 (1980); K. Suzuki and S.Y. Lee, Prog. Theor. Phys. 64, $2091(1980)$

[15] S.K. Bogner, T.T.S. Kuo, and A. Schwenk, Phys. Rep. 386, 1 (2003)

[16] S.K. Bogner, R.J. Furnstahl, and R.J. Perry, Phys. Rev. C 75, 061001 (2007)

[17] G. Hagen, D.J. Dean, M. Hjorth-Jensen, T. Papenbrock, and A. Schwenk, Phys. Rev. C (in press, 2007)

[18] S.K. Bogner, A. Schwenk, R.J. Furnstahl, and A. Nogga, Nucl. Phys. A 763, 59 (2005).

[19] A. Nogga, S.K. bogner, A. Schwenk, Phys. Rev. C 70, 061002 (2004)

[20] S.C. Pieper and R.B. Wiringa, Ann. Rev. Nucl. Part. Sci. 51, 53 (2001)

[21] P. Navratil, J.P. Vary, and B.R. Barrett, Phys. Rev. C 62, 054311 (2000)

[22] S.C. Pieper, V.R. Pandharipande, R.B. Wiringa, and J. Carlson, Phys. Rev. C 64, 014001 (2001)

[23] K.M. Nollett, S.C. Pieper, R.B. Wiringa, J. Carlson, and G.M. Hale, Phys. Rev. Lett. 99, 022502 (2007)

[24] P. Navratil, C.A. Bertulani, and E. Caurier, Phys. Rev. C 73, 065801 (2006)

[25] A. Deltuva and A.C. Fonseca, Phys. Rev. C 76, 021001(R) (2007)

[26] F. Coester, Nucl. Phys. 7, 421 (1958); F. Coester and H. Kümmmel, Nucl. Phys. 17, 477 (1960)

[27] H. Kümmel, K.H. Lührmann, and J.G. Zabolitzky, Phys. Rep. 36, 1 (1978)

[28] J. Cížek, J. Chem. Phys. 45, 4256 (1966)

[29] J.H. Heisenberg and B. Mihaila, Phys. Rev. C 59, 1440 (1999)

[30] D.J. Dean and M. Hjorth-Jensen, Phys. Rev. C 69, 054320 (2004)

[31] K. Kowalski, D.J. Dean, M. Hjorth-Jensen, T. Papenbrock, and P. Piecuch, Phys. Rev. Lett. 92, $132501(2004)$

[32] G. Hagen, D.J. Dean, M. Hjorth-Jensen, and T. Papenbrock, Phys. Lett. B, in press (2007)

[33] G. Hagen, T. Papenbrock, D.J. Dean, A. Schwenk, A. Nogga, M. Wloch, and P. Piecuch, Phys. Rev. C (in press, 2007)

[34] R.J. Bartlett and M. Musia, Rev. Mod. Phys. 79, 291 (2007)

[35] N. Michel, W. Nazarewicz, M. Płoszajczak, and K. Bennaceur, Phys. Rev. Lett. 89, 042502 (2002)

[36] R. Id Betan, R.J. Liotta, N. Sandulescu, and T. Vertse, Phys. Rev. Lett. 89, 042501 (2002).

[37] G. Hagen, M. Hjorth-Jensen, and N. Michel, Phys. Rev. C 73, 064307 (2006).

[38] T. Bergren, Nucl. Phys. A 109265 (1968)

[39] A. Volya and V. Zelevinsky, Phys. Rev. Lett. 94, 052501 (2005)

[40] I. Rotter, Rep. Prog. Phys. 54, 635 (1991).

[41] J. Okołowicz, M. Płoszajczak, and I. Rotter, Phys. Rep. 374, 271 (2003)

[42] G. Audi, A.W. Wapstra, and C. Thibault, Nucl. Phys. A 729, 3 (2003)

[43] M.V. Stoitsov, J. Dobaczewski, W. Nazarewicz, S. Pittel, and D.J. Dean, Phys. Rev. C 68, 054312 (2003) 
[44] M. Samyn, S. Goriely, and J.M. Pearson, Phys. Rev. C 72, 044316 (2005) 
This figure "figure1.jpg" is available in "jpg" format from: http://arxiv.org/ps/0709.0441v1 